Publ. RIMS, Kyoto Univ.

14 (1978), 337-358

\title{
Theory of Division Games
}

- Dedicated to the memory of Professor Taira Honda-

By

Yōhei YAMASAKI*

\begin{abstract}
Lehman [9] generalized the Shannon's switching games to those on Boolean functions. We discuss in this paper, the theory of division games which contain Lehman's games and their reverse games. The theory of Lehman's games is analyzed and rearranged in this paper. Our results for division games contain naturally those for the classical ones as Hex, Bridg-it and their reverse games. Especially for the reverse games, we introduce several concepts dual to those introduced before, and obtain concise reformulations.

The most significant feature of this paper is that we adopt the notion "assignment" to deal with generalized games in which the principle of alternate move does not necessarily hold. The classical results will be accordingly purified and divided into four theorems, i. e., Theorems 2, 3, 4 and 5, which indicate the dualities about points and about assignments, with respect to signature and to players. It is also a virtue of this generalization that we can characterize in a game theoretical argument the triple systems and the block designs $W_{11}$ and $W_{12}$ whose automorphism groups are Mathieu groups $M_{11}$ and $M_{12}$.
\end{abstract}

\section{Introduction}

Consider Hex. Two players, say White and Black play on a $\mathrm{Hex}$ board consisting of $n$ rows and $n$ columns of hexagons arranged in a rhombus. We colour each pair of opposite side of edges white and black respectively. They play alternately, placing a counter of their colour on an unoccupied hexagon. Each player wins if he forms a chain of adjacent counters that joins his side of the board to the opposite side. A game in case $n=4$ is illustrated in Figure 1, where Black has won.

Communicated by S. Hitotumatu, February 14, 1977.

* Department of Mathematics, Osaka University, Toyonaka, Osaka 560, Japan. 


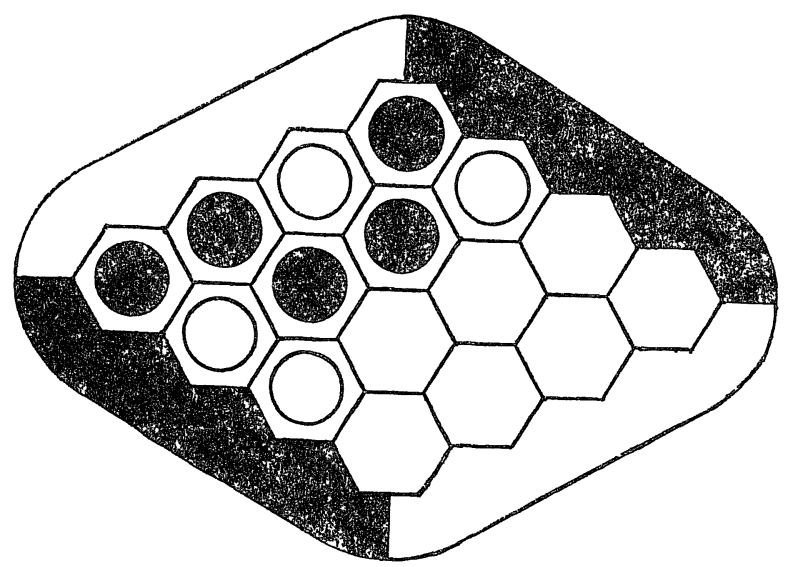

Figure 1

For every $n$ the game of Hex has the following properties:

$\left(\mathbf{P}_{1}\right)$ No draw is possible,

$\left(\mathbf{P}_{2}\right) \quad$ There exists a winning strategy for the player who moves first.

In Sections 1 and 2, we prepare several terminologies on division spaces, extending Lehman's idea. A division space is defined as a pair of a finite set and a decision of winners, just like, in Hex, a Hex board yields the set of hexagons and a winner can be decided by a final position of hexagons occupied entirely. Here we give the definition of a division space. Let a set $I$ of two players $T$ and $\perp$ be given. Let $X$ be a finite set. Then we call a mapping from $X$ to $\Pi$ a division on $X$. The set of divisions on $X$ is denoted by $\mathfrak{I}_{X}$. Let $\chi^{*}$ be a mapping from $\mathfrak{D}_{x} \times \Pi$ to $\{-1,1\}$ such that

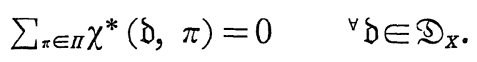

Then we call the pair $\mathscr{D}^{*}\left(X, \chi^{*}\right)$ a division space. One can characterize the division space for Hex by some properties defined in general on division spaces.

After these sections, we study various games played on division spaces, where the moves are not necessarily made alternately. Our situation is as follows. Let $\mathscr{D}^{*}=\left(X, \chi^{*}\right)$ be a division space. Then a mapping from the set $\mathbb{N}_{|X|}$ of positive integers not exceeding the number $|X|$ of elements of $X$ to $\Pi$ is called an assignment over $\mathscr{D}^{*}$. 
A pair $\Gamma=\left(\mathscr{D}^{*}, a\right)$ of a division space $\mathscr{D}^{*}$ and an assignment $a$ over it is called a division game. The division game $\Gamma$ is played in the following way. For each number $i$ of $N_{|x|}$, the player $a(i)$ occupies an unoccupied point on $X$, at the $i$-th stage. At the final stage the points are occupied entirely and we regard a division b has occured, where $\mathfrak{D}$ is given by

$$
\mathfrak{D}(x)=\pi \text { iff } x \text { is occupied by } \pi .
$$

Then the player $\pi$ such that $\chi^{*}(\delta, \pi)=1$ is regarded as the winner. We prepare an induction theorem and four other duality theorems, which cause us to generalize the results concerning winning strategies for classical games. Here we give Theorem 4, the key theorem of this paper, in a colloquial style.

Theorem 4. Let $\mathscr{D}^{*}=\left(X, \chi^{*}\right)$ be a division space with $X$ nonempty and $a$ and $a^{\prime}$ assignments over it such that

$$
a(|X|)=a^{\prime}(1)
$$

and

$$
a(i)=a^{\prime}(i+1) \quad 1 \leqq{ }^{\forall} i<|X| .
$$

Suppose that $a(|X|)$ has a winning strategy for the division game $\left(\mathscr{D}^{*}, a\right)$. Then he has one for the division game $\left(\mathscr{D}^{*}, a^{\prime}\right)$.

In the forthcoming paper we shall discuss the graph theory of generalized games of Hex and Bridg-it, by a different approach from that of Shannon's switching games. We shall define a class of graphs called "connex" and study the possibility of embedding it into a manifold.

Now we mention here the history of division games. The idea of division games was first seen in Lehman [9]. He has remarked briefly a fact concerning with the games over regular division spaces along alternate assignments in our terminology cf. Section 7. This idea is developed by Yamasaki [11]. After several discussions with Mr. Masahiko Sato of RIMS, Kyoto University*, the author

* His present address is Dept. of Math., College of General Education, University of Tokyo. 
presented a wider class of games containing Hex, which he named division games, and stated several propositions. Mr. Sato appreciated these results and was interested in the theory. They agreed in a view that there should exist a tendency to win or to lose for a player in certain types of division games. Some of the formulations were improved by Sato [10]. For instance, he suggested the author the definition of divisions in the present style and the idea to calculate the value of judge. After these improvements the author completed the duality theorems and gave new proofs in arithmetic arguments.

The author thanks Mr. Sato for his valuable suggestions and his contribution to found the theory of division games.

\section{§1. Division Space}

As usual "iff" means "if and only if". We denote by $\boldsymbol{Z}, \bar{N}$ and $N$ the set of integers, that of non-negative integers and that of positive integers, respectively. We also denote by $\boldsymbol{N}_{n}$ the set of positive integers not exceeding $n$ for $n \in \bar{N}$. Let $\bar{\Pi}$ be a set consisting of three elements $T, \perp$ and $\theta$. We call $T$ and $\perp$ the players and $\theta$ nobody. $\Pi$ denotes the subset of $\bar{\Pi}$ consisting of two players. An element of $\Pi$ in general is denoted by $\pi$. We denote by $\wedge$ the involution of $\Pi$ without fixed points, i. e., $\hat{T}=\perp$ and $\hat{I}=T$.

For any sets $X$ and $Y$ we denote by $[X \rightarrow Y]$ the set of mappings from $X$ to $Y$ and by $|X|$ or $\# X$ the number of elements of $X$ when $X$ is a finite set.

Let $X$ be a finite set. We set $\mathfrak{D}_{X}=[X \rightarrow \Pi]$ and $\mathfrak{P}_{x}=[X \rightarrow \bar{\Pi}]$. An element $\mathfrak{b}$ of $\mathfrak{D}_{X}$ is called a division, and one $\partial$ of $\mathfrak{P}_{X}$ is called a position on $X$. A mapping $\chi^{*}: \mathscr{D}_{X} \times \Pi \rightarrow\{-1,1\}$ is called a iudge on $X$ if

$$
\sum_{\pi \in \Pi} \chi^{*}(\mathscr{D}, \pi)=0 \quad \forall \delta \in \mathscr{D}_{\mathbf{x}} .
$$

A pair $\mathscr{D}^{*}=\left(X, \chi^{*}\right)$ is called a division space. The letter $X$ may be omitted while it is fixed.

Let $\mathscr{D}^{*}=\left(X, \chi^{*}\right)$ be a division space, $\partial$ a position on $X$ and $\partial^{\prime}$ a position on $\partial^{-1}(\theta)$. Then we compose a position $\partial \cup \partial^{\prime}$ on $X$ by 
the following equation:

$$
\left(\partial \cup \partial^{\prime}\right)^{-1}(\pi)=\partial^{-1}(\pi) \cup \partial^{\prime-1}(\pi) \quad{ }^{\forall} \pi \in \Pi .
$$

If $\mathfrak{D}_{\partial}$ is a division on $\partial^{-1}(\theta)$, then $\partial \cup \grave{D}_{\partial}$ is a division on $X$.

Proposition 1. Let $\mathscr{D}^{*}=\left(X, \chi^{*}\right)$ be a division space and $\partial$ a position on $X$. We define a pair $\mathscr{D}_{0}^{*}=\left(X_{\partial}, \chi_{0}^{*}\right)$ of a finite set $X_{\partial}$ and a mapping $\chi_{\partial}^{*}: \mathfrak{D}_{x_{\partial}} \times \Pi \rightarrow\{1,-1\}$ as follows:

$$
\begin{aligned}
& X_{\partial}=\partial^{-1}(\theta) \\
& \chi_{\partial}^{*}\left(\mathfrak{D}_{\partial}, \pi\right)=\chi^{*}\left(\partial \cup \mathfrak{D}_{\partial}, \pi\right) \quad{ }^{\forall} \mathfrak{D}_{\partial} \in \mathscr{D}_{X_{\partial}} .
\end{aligned}
$$

Then $\mathscr{D}_{a}^{*}$ is a division space.

The proof is easy. The division space $\mathscr{D}_{0}^{*}$ corresponds to the situation where each player $\pi$ has occupied the subset $\partial^{-1}(\pi)$ of $X$.

Let $\mathscr{D}^{*}=\left(X, \chi^{*}\right)$ be a division space. Then $\mathscr{D}^{*}$ is said to be regular if

$$
\mathfrak{D}_{1}^{-1}(\pi) \supset \mathfrak{D}_{2}^{-1}(\pi) \text { implies } \chi^{*}\left(\mathfrak{D}_{1}, \pi\right) \geqq \chi^{*}\left(\mathfrak{D}_{2}, \pi\right) \text {, }
$$

misère if

$$
\mathfrak{D}_{1}^{-1}(\pi) \supset \mathfrak{D}_{2}^{-1}(\pi) \text { implies } \chi^{*}\left(\wp_{1}, \pi\right) \leqq \chi^{*}\left(\bigvee_{2}, \pi\right),
$$

and trivial if

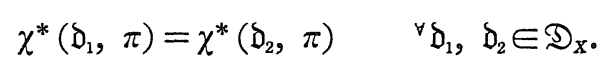

When $\mathscr{D}^{*}$ is regular (misère or trivial) the symbol * may be replaced by ${ }^{+}\left({ }^{-}\right.$or $\left.{ }^{0}\right)$. A subset $S$ of $X$ is said to be regular, misère or negligible if $\mathscr{D}_{\partial}^{*}$ is regular, misère or trivial for any position $\partial$ on $X$ such that

$$
\partial^{-1}(\theta)=S
$$

A point $x$ of $X$ is said to be regular, misère or negligible if $\{x\}$ is so. We denote by $X^{+}, X^{-}$and $X^{0}$ the set of regular points, that of misère points and that of negligible points of $X$.

Proposition 2. Let $\mathscr{D}^{*}=\left(X, \chi^{*}\right)$ be a division space. Then $X^{+}$ $\left(X^{-}\right.$or $\left.X^{0}\right)$ is the maximal regular (misère or negligible, respectively) 
subset of $X$, and

$$
X^{0}=X^{+} \cap X^{-}
$$

Proposition 3. Let $\mathscr{D}^{*}=\left(X, \chi^{*}\right)$ be a division space. Then $\mathscr{D}^{*}$ is regular or misère if and only if the set $X$ itself is so, and $\mathscr{D}^{*}$ is trivial if and only if $X$ is negligible.

These propositions are easily verified and their proofs are omitted.

Here we give a brief comment on the correspondence between Hex and division spaces. Hex game often finishes with many hexagons unoccupied. But a game can be continued, until the hexagons are occupied entirely. The winner remains unchanged even if the decision is made at the final position. So we can regard a Hex game to be finished when all hexagons are occupied. Now let $X$ be the set of hexagons on the Hex board and let $\chi^{+}$be the mapping which sends each pair ( $\delta, \pi)$ to 1 if $\pi$ is the winner at the final position where $\pi$ has occupied $\mathfrak{D}^{-1}(\pi)$, and to -1 if he is the loser. Then it is clear that $\mathscr{H}_{n}^{+}=\left(X, \chi^{+}\right)$is a regular division space. Similarly for Reverse Hex, we naturally have a division space $\mathscr{H}_{n}^{-}=$ $\left(X, \chi^{-}\right)$.

\section{$\S 2$. Homomorphism}

Let $\mathscr{D}^{*}$ and $\mathscr{D}_{\imath}^{*}=\left(X_{\imath}, \chi_{\imath}^{*}\right)_{(i=1,2)}$ be division spaces. A pair $f=$ $\left(f^{x}\right.$, sgn $f$ ) consisting of a mapping $f^{x}: X_{1} \rightarrow X_{2}$ and a permutation sgn $f$ of $\Pi$ is said to be a pseudo-homomorphism if the following diagram commutes :

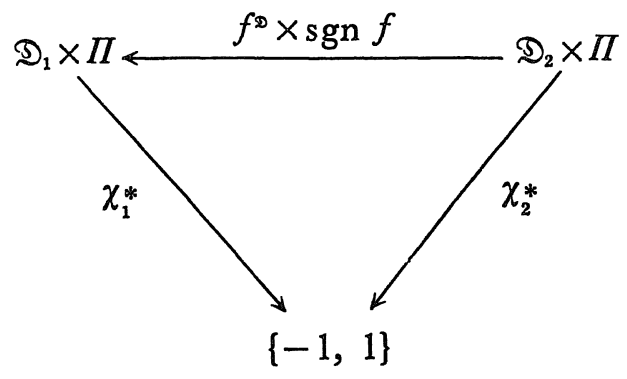


where $f^{\triangleright}$ is given by

$$
f^{\triangleright}\left(\mathfrak{D}_{2}\right)=\operatorname{sgn} f \circ \mathfrak{D}_{2} \circ f^{x} .
$$

A pseudo-homomorphism $f=\left(f^{x}, \operatorname{sgn} f\right)$ is said to be a pseudo-immersion if $f^{x}$ is injective, a pseudo-contraction if $f^{x}$ is surjective, and a pseudo-isomorphism if $f^{x}$ is bijective. A pseudo-isomorphism $f$ from $\mathscr{D}^{*}$ to $\mathscr{D}^{*}$ is called a pseudo-automorphism. The prefix "pseudo-" is omitted if $\operatorname{sgn} f=\mathrm{id}_{n}$ and replaced by "anti-" if sgn $f=\wedge$. The set $\mathscr{A}_{u t} \mathscr{D}^{*}$ of pseudo-automorphisms naturally admits a group structure. The set $\mathscr{A}_{u t} \mathscr{D}^{*}$ of automorphisms is a normal subgroup of $\mathscr{A}_{u t} \mathscr{D}^{*}$ of index 1 or 2 . They are called the pseudo-automorphism group and the automorphism group of $\mathscr{D}^{*}$. A division space $\mathscr{D}^{*}=$ $\left(X, \chi^{*}\right)$ is said to be impartial if the index above is 2, and strongly impartial if $\left(\mathrm{id}_{\mathrm{x}}, \widehat{\widehat{N}}\right)$ is an anti-automorphism.

The theory of Hex and Reverse Hex will be developed in that of regular impartial division spaces and that of misère impartial ones. Furthermore there is an analogous theory in case $\mathscr{D}^{*}$ is impartial, even if $\mathscr{D}^{*}$ is neither regular nor misère.

\section{§3. Division Game}

From now on we study various games played over a division space, where the moves are not necessarily made alternately. Our statements are often expressed using double signs \pm and $\mp$. They take the upper signs or the lower signs consistently.

For $\mathrm{N} \in \bar{N}$, an element of $\left[\mathbb{N}_{N} \rightarrow \Pi\right]$ is called an assignment of length $N$. Let $\mathscr{D}^{*}=\left(X, \chi^{*}\right)$ be a division space. Then an assignment of length $|X|$ is called one over $\mathscr{D}^{*}$, also. Let $a$ be an assignment over $\mathscr{D}^{*}$. Then the pair $\Gamma=\left(\mathscr{D}^{*}, a\right)$ is called a division game. Now the division game $\Gamma$ is played by the two players $T$ and $\perp$ as follows :

i) $\quad a(i)$ is assigned to make the $i$-th move,

ii) $a(i)$ occupies an unoccupied point as the $i$-th move,

iii) after the last (i. e., the $|X|$-th) move is made, we consider that a division $\delta$ occurs and that $\pi$ wins if $\chi^{*}(\delta, \pi)=1$ 
where the division $\mathfrak{b}: X \rightarrow \Pi$ is defined by

$$
\mathfrak{D}(x)=\pi \text { iff } \pi \text { has occupied } x .
$$

As in the theory of finite games we easily see that for any division game $\Gamma$ there exists a player who has a winning strategy.

Let $\Gamma$ be a division game. Then we define two functions $\omega^{+}$ and $\omega^{-}$by

$$
\omega^{+}(\Gamma, \pi)=-\omega^{-}(\Gamma, \pi)=\left\{\begin{array}{r}
1 \text { if } \pi \text { has a winning strategy } \\
-1 \text { if } \hat{\pi} \text { has a winning strategy. }
\end{array}\right.
$$

Let $S_{\mathrm{T}}$ and $S_{\perp}$ be arbitrary subsets of $X$ with $S_{\mathrm{T}} \cap S_{\perp}=\varnothing$. Then we define a position $\partial$ as follows:

$$
\partial(x)=\left\{\begin{array}{l}
\top \text { if } x \in S_{\top} \\
\perp \text { if } x \in S_{\perp} \\
\theta \text { otherwise }
\end{array}\right.
$$

This position is denoted by $\sum_{\pi \in \Pi} S_{\pi} \cdot \pi,\left(S_{T} \cdot T+S_{\perp} \cdot \perp\right)$. In the above expressions, $S_{\pi}$ can be replaced by $x$ if $S_{\pi}$ consists of exactly one point $x$, and $S_{\pi} \circ \pi$ can be omitted if $S_{\pi}$ is empty.

We define the permutation $s_{N}$ of $N_{N}$ for $N \in N$ by

$$
\begin{array}{ll}
s_{N}(i)=i+1 & 1 \leqq \\
s_{N} & (N)=1 . N-1
\end{array}
$$

Let $a$ be an assignment of length $N$ with $N \in \mathbb{N}$. Then we put $a_{+k}=\left.a\right|_{N_{N-k}}$ and $a_{-k}=\left.\left(a \circ s_{N}^{k}\right)\right|_{N_{N-k}}$ for $k \in N_{N}$. We put also $a_{ \pm}=a_{ \pm 1}$. When $N \geqq 1$, the player $a(1)$ is called the starting player and denoted by $\pi_{+}(a)$. The player $a(N)$ is called the closing player and denoted by $\pi_{-}(a)$. When $N \geqq 2$, we simply write $a_{+-}$and $a_{-+}$instead of $\left(a_{+}\right)_{-}$ and $\left(a_{-}\right)+$respectively.

\section{§ 4. Induction Theorem}

Lemma 1. Let $X$ be a finite set with $|X| \geqq 2$ and $f$ a mapping from $X \times X$ to $\{-1,1\}$ such that

$$
f(x, y)=f(y, x) \quad{ }^{\forall} x, y \in X .
$$

Then we have 


$$
\min _{x \in X} \max _{y \in X-\{x\}} f(x, y) \geqq \max _{y \in X} \min _{x \in X-\{y\}} f(x, y) .
$$

Proof. We fix pairs $\left(x_{1}, y_{1}\right)$ and $\left(x_{2}, y_{2}\right)$ with $x_{i} \neq y_{i}$ for each $i$ where $f$ attains the mini-max value and the maxi-min value. First suppose $x_{1} \neq y_{2}$. Then we have

$$
f\left(x_{1}, y_{1}\right) \geqq f\left(x_{1}, y_{2}\right) \geqq f\left(x_{2}, y_{2}\right) .
$$

Next suppose $x_{1}=y_{2}$. Then we have

$$
f\left(x_{1}, y_{1}\right)=f\left(y_{2}, y_{1}\right)=f\left(y_{1}, y_{2}\right) \geqq f\left(x_{2}, y_{2}\right),
$$

which proves our lemma.

Theorem 1. Let $\Gamma=\left(\mathscr{D}^{*}, a\right)=\left(X, \chi^{*}, a\right)$ be a division game with $|X| \neq 0$. Then we have the followings:

$$
\begin{array}{ll}
(+) & \omega^{+}\left(\mathscr{D}^{*}, a, \pi\right)=\max _{x \in X} \omega^{+}\left(\mathscr{D}_{x \cdot \pi}^{*}, a_{-}, \pi\right) \text { if } \pi_{+}(a)=\pi, \\
(-) & \omega^{-}\left(\mathscr{D}^{*}, a, \pi\right) \geqq \max _{x \in X} \omega^{-}\left(\mathscr{D}_{x \cdot \pi}^{*}, a_{+}, \pi\right) \text { if } \pi_{-}(a)=\pi .
\end{array}
$$

Proof. The first assertion (+) is evident since it is just an inductive definition of $\omega^{+}$. We shall prove the second assertion (-) by induction on $|X|$.

Step 1. When $|X|=1$, the assertion is evident.

Step 2. We assume that the assertion is valid for $|X|-1$. First suppose that $\pi_{+}(a)=\hat{\pi}$. Then by the induction hypothesis and the assertion $(+)$, we have

$$
\begin{aligned}
& \max _{x \in X} \omega^{-}\left(\mathscr{D}_{x \cdot \pi}^{*}, a_{+}, \pi\right) \\
= & \max _{x \in X} \max _{y \in X-\{x\}} \omega^{-}\left(\mathscr{D}_{x \cdot \pi+y \cdot \pi}^{*}, a_{+-}, \pi\right) \\
= & \max _{y \in X} \max _{x \in X-\{y\}} \omega^{-}\left(\left(\mathscr{D}_{y \cdot \pi}\right)_{x \cdot \pi}^{*}, a_{-+}, \pi\right) \\
\leqq & \max _{y \in X} \omega^{-}\left(\mathscr{D}_{y \cdot \pi}^{*}, a_{-}, \pi\right) \\
= & \omega^{-}\left(\mathscr{D}^{*}, a, \pi\right)
\end{aligned}
$$

Next suppose that $\pi_{+}(a)=\pi$. Then by the induction hypothesis, the assertion $(+)$ and Lemma 1 ,

$$
\begin{aligned}
& \max _{x \in X} \omega^{-}\left(\mathscr{D}_{x \cdot \pi}^{*}, a_{+}, \pi\right) \\
= & \max _{x \in X} \min _{y \in X-\{x\}} \omega^{-}\left(\mathscr{D}_{\{x, y\} \cdot \pi}^{*}, a_{+-}, \pi\right) \\
\leqq & \min _{y \in X} \max _{x \in X-\{y\}} \omega^{-}\left(\mathscr{D}_{\{x, y\} \cdot \pi}^{*}, a_{-+}, \pi\right) \\
\leqq & \min _{y \in X} \omega^{-}\left(\mathscr{D}_{y \cdot \pi}^{*}, a_{-}, \pi\right) \\
= & \omega^{-}\left(\mathscr{D}^{*}, a, \pi\right) .
\end{aligned}
$$


Now our proof is accomplished.

\section{§5. Duality Theorems}

Theorem 2. Let $\mathscr{D}^{*}=\left(X, \chi^{*}\right)$ be a division space with $|X| \neq 0$, $x$ and $y$ be elements of $X^{ \pm}$and $a$ an assignment of length $|X|-1$. Then we have

$$
\omega^{ \pm}\left(\mathscr{D}_{x}^{*} \cdot \mathrm{T}, a, \mathrm{~T}\right)+\omega^{ \pm}\left(\mathscr{D}_{y \cdot \perp}^{*}, a, \perp\right) \geqq 0,
$$

in other words :

$$
\omega^{ \pm}\left(\mathscr{D}_{x \cdot \pi}^{*}, a, \pi\right) \geqq \omega^{ \pm}\left(\mathscr{D}_{y \cdot \hbar}^{*}, a, \pi\right) \quad{ }^{\forall} \pi \in \Pi .
$$

Proof. We shall prove this assertion by induction on $|X|$.

Step 1. If $|X|=1$, then the assertion is easily seen.

Step 2. We assume that the assertion is valid for $|X|-1$.

If $x=y$ the proof is easy. Then we have to see the assertion only in case where $x \neq y$. It suffices to show the assertion in case $\pi_{+}(a)=\pi$.

Case $(+)$. By the induction hypothesis, Theorem 1 and the fact that $x, y \in X^{+}$we have

$$
\begin{aligned}
& \omega^{+}\left(\mathscr{D}_{x \cdot \pi}^{*}, a, \pi\right) \\
= & \max _{z \in X-\{x\}} \omega^{+}\left(\mathscr{D}_{\{x, z\} \cdot \pi}^{*}, a_{-}, \pi\right) \\
= & \max \left\{\max _{z \in X-\{x, y\}} \omega^{+}\left(\mathscr{D}_{\{x, z\} \cdot \pi}^{*}, a_{-}, \pi\right), \omega^{+}\left(\mathscr{D}_{\{x, y\} \cdot \pi}^{*}, a_{-}, \pi\right)\right\} \\
\geqq & \max \left\{\max _{z \in X-\{x, y\}} \omega^{+}\left(\mathscr{D}_{z \cdot \pi+y \cdot \pi}^{*}, a_{-}, \pi\right), \omega^{+}\left(\mathscr{D}_{x \cdot \pi+y \cdot \pi}^{*}, a_{-}, \pi\right)\right\} \\
= & \max _{z \in X-\{y\}} \omega^{+}\left(\left(\mathscr{D}_{y \cdot \pi}\right)_{z \cdot \pi}^{*}, a_{-}, \pi\right) \\
= & \omega^{+}\left(\mathscr{D}_{y \cdot \pi}^{*}, a, \pi\right) .
\end{aligned}
$$

Case (-). The induction hypothesis, Theorem 1 and the fact that $x, y \in X^{-}$give us

$$
\begin{aligned}
& \omega^{-}\left(\mathscr{D}_{x \cdot \pi}^{*}, a, \pi\right) \\
= & \min _{z \in X-\{x\}} \omega^{-}\left(\mathscr{D}_{\{x, z\} \cdot \pi}^{*}, a_{-}, \pi\right) \\
= & \min \left\{\min _{z \in X-\{x, y\}} \omega^{-}\left(\mathscr{D}_{\{x, z\} \cdot \pi}^{*}, a_{-}, \pi\right), \omega^{-}\left(\mathscr{D}_{\{x, y\} \cdot \pi}^{*}, a_{-}, \pi\right)\right\} \\
\geqq & \min \left\{\min _{z \in X-\{x, y\}} \omega^{-}\left(\mathscr{D}_{y \cdot t+z \cdot \pi}^{*}, a_{-}, \pi\right), \omega^{-}\left(\mathscr{D}_{y \cdot \pi+x \cdot \pi}^{*}, a_{-}, \pi\right)\right\} \\
= & \min _{z \in X-\{y\}} \omega^{-}\left(\mathscr{D}_{y \cdot \pi+z \cdot \pi}^{*}, a_{-}, \pi\right) \\
= & \omega^{-}\left(\mathscr{D}_{y \cdot \pi}^{*}, a, \pi\right) .
\end{aligned}
$$

Now our proof is completed. 
Theorem 3. Let $\mathscr{D}^{*}=\left(X, \chi^{*}\right)$ be a division space with $\mid X_{j} \neq 0$, $x$ a regular point, $y$ a misère point in $X$ and $a$ an assignment of length $|X|-1$. Then we have

$$
\omega^{+}\left(\mathscr{D}_{x \cdot \pi}^{*}, a, \pi\right)+\omega^{-}\left(\mathscr{D}_{y \cdot \pi}^{*}, a, \pi\right) \geqq 0 \quad{ }^{\forall} \pi \in \Pi \text {. }
$$

Proof. We give the proof of the assertion by induction on $|X|$. It is easy to see that this assertion is equivalent to

$$
\omega^{+}\left(\mathscr{D}_{y \cdot \pi}^{*}, a, \pi\right) \leqq \omega^{+}\left(\mathscr{D}_{x \cdot \pi}^{*}, a, \pi\right) \quad{ }^{\forall} \pi \in \Pi \text {. }
$$

It suffices to prove this assertion only in case $x \neq y$.

Step 1. If $|X|=1$, the proof is easy.

Step 2. We assume the assertion is valid for $|X|-1$.

Case $\pi_{+}(a)=\pi$. We have

$$
\begin{aligned}
& \omega^{+}\left(\mathscr{D}_{x \cdot \pi}^{*}, a, \pi\right) \\
= & \max _{z \in X-\{x\}} \omega^{+}\left(\mathscr{D}_{\{x, \pi\} \cdot \pi}^{*}, a_{-}, \pi\right) \\
= & \max \left\{\max _{z \in X-\{x, y\}} \omega^{+}\left(\mathscr{D}_{\{x, x\} \cdot \pi}^{*}, a_{-}, \pi\right), \omega^{+}\left(\mathscr{D}_{\{x, y\} \cdot \pi}^{*}, a_{-}, \pi\right)\right\} \\
\geqq & \left.\max _{\left\{\max _{z} \in X-\{x, y\}\right.} \omega^{+}\left(\mathscr{D}_{\{z, y\} \cdot \pi}^{*}, a_{-}, \pi\right), \omega^{+}\left(\mathscr{D}_{\{x, y\} \cdot \pi}^{*}, a_{-}, \pi\right)\right\} \\
= & \max _{2 \in X-\{y\}} \omega^{+}\left(\mathscr{D}_{\{y, z\} \cdot \pi}^{*}, a_{-}, \pi\right) \\
= & \omega^{+}\left(\mathscr{D}_{y \cdot \pi}^{*}, a, \pi\right),
\end{aligned}
$$

by induction hypothesis and Theorem 1.

Case $\pi_{+}(a)=\hat{\pi}$. We have

$$
\begin{aligned}
& \omega^{+}\left(\mathscr{D}_{x \cdot \pi}^{*}, a, \pi\right) \\
= & \min _{z \in X-\{x\}} \omega^{+}\left(\mathscr{D}_{x \cdot \pi+z \cdot \pi}^{*}, a_{-}, \pi\right) \\
= & \min \left\{\min _{z \in X-\{x, y\}} \omega^{+}\left(\mathscr{D}_{x \cdot \pi+z \cdot \pi}^{*}, a_{-}, \pi\right), \omega^{+}\left(\mathscr{D}_{x \cdot \pi+y \cdot \pi}^{*}, a_{-}, \pi\right)\right\} \\
\geqq & \min \left\{\min _{z \in X-\{x, y\}} \omega^{+}\left(\mathscr{D}_{y \cdot \pi+z \cdot \pi}^{*}, a_{-}, \pi\right), \omega^{+}\left(\mathscr{D}_{y \cdot \pi+x \cdot \hbar}^{*}, a_{-}, \pi\right)\right\} \\
= & \min _{z \in X-\{y\}} \omega^{+}\left(\mathscr{D}_{y \cdot \pi+z \cdot \pi}^{*}, a_{-}, \pi\right) \\
= & \omega^{+}\left(\mathscr{D}_{y \cdot \pi}^{*}, a, \pi\right),
\end{aligned}
$$

by induction hypothesis, Theorem 1 and the facts $x \in X^{+}$and $y \in X^{-}$. Now our proof is accomplished.

Theorem 4. Let $\mathscr{D}^{*}=\left(X, \chi^{*}\right)$ be a division space and $a$ an assignment of length $|X|+1$ with $\pi_{+}(a)=\pi_{-}(a)$. Then we have

$$
\omega^{+}\left(\mathscr{D}^{*}, a_{+}, \pi\right)+\omega^{-}\left(\mathscr{D}^{*}, a_{-}, \pi\right) \geqq 0
$$


if $\pi_{+}(a)=\pi_{-}(a)=\pi_{\text {. }}$

Proof. The proof is easy when $|X|=0$. We suppose $|X| \neq 0$. Then we have

$$
\begin{aligned}
& \omega^{+}\left(\mathscr{D}^{*}, a_{+}, \pi\right) \\
= & \max _{x \in \mathrm{x}} \omega^{+}\left(\mathscr{D}_{x \cdot \pi}^{*}, a_{+--}, \pi\right) \\
\geqq & \min _{x \in \mathrm{x}} \omega^{+}\left(\mathscr{D}_{x \cdot \pi}^{*}, a_{-+}, \pi\right) \\
\geqq & \omega^{+}\left(\mathscr{D}^{*}, a_{-}, \pi\right)
\end{aligned}
$$

by Theorem 1. This inequality is equivalent to what we have to prove.

Proposition 4. Let $\mathscr{D}^{*}=\left(X, \chi^{*}\right)$ be a division space with $|X|$ $\neq 0$ and let $a$ be an assignment over $\mathscr{D}^{*}$. Then

$$
\omega^{+}\left(\mathscr{D}^{*}, a, \pi_{+}(a)\right) \geqq \omega^{+}\left(\mathscr{D}^{*}, a \circ s_{|X|}, \pi_{+}(a)\right) .
$$

Proof. We easily find an assignment $a^{\prime}$ of length $|X|+1$ such that $a_{+}^{\prime}=a$ and $a_{-}^{\prime}=a \circ s_{|x|}$. Now the assertion follows easily by the previous theorem.

\section{§6. Game Theory over a Regular or Misère Space}

In this section we deal with a regular or misère division space only.

Theorem 5. Let $\mathscr{D}^{ \pm}=\left(X, \chi^{ \pm}\right)$be a regular (misère) division space and let $a$ and $a^{\prime}$ be assignments over it satisfying

$$
a(n)=\top \text { or } a^{\prime}(n)=\perp \quad{ }^{\forall} n \in N_{|X|} \cdot
$$

Then we have

$$
\omega^{ \pm}\left(\mathscr{D}^{ \pm}, a, \top\right)+\omega^{ \pm}\left(\mathscr{D}^{ \pm}, a^{\prime}, \perp\right) \geqq 0 .
$$

Proof. We have only to show this assertion when $a(n)$ coincides with $a^{\prime}(n)$ for each $n \in N_{|x|}$ with a sole exception. In such a case the assertion follows by Theorem 2 in an inductive argument. 
From the previous theorem and Theorem 1, we easily obtain the following proposition.

Proposition 5. Let $\mathscr{D}^{ \pm}=\left(X, \chi^{ \pm}\right)$be a regular (misère) division space and let $a$ be an assignment over it. Let $S$ be a subset of $X$. Then we have

$$
\omega^{ \pm}\left(\mathscr{D}_{S}^{ \pm} \cdot \pi, a_{ \pm|s|}, \pi\right) \geqq \omega^{ \pm}\left(\mathscr{D}^{ \pm}, a, \pi\right) .
$$

Especially if $S$ is negligible, then for each player $\pi$

$$
\omega^{ \pm}\left(\mathscr{D}_{s}^{ \pm}, a_{ \pm|s|}, \pi\right)=\omega^{ \pm}\left(\mathscr{D}^{ \pm}, a, \pi\right) .
$$

Proof. It suffices to show the assertion only in case $|S|=1$.

Case $\pi_{\mp}(a)=\pi$. By the previous theorem and Theorem 1, we have

$$
\begin{aligned}
& \omega^{ \pm}\left(\mathscr{D}^{ \pm}, a, \pi\right) \\
\leqq & \min _{x \in X} \omega^{ \pm}\left(\mathscr{D}_{x \cdot \pi}^{ \pm}, a_{ \pm}, \pi\right) \\
\leqq & \omega^{ \pm}\left(\mathscr{D}_{s \cdot \pi}^{ \pm}, a_{ \pm}, \pi\right) .
\end{aligned}
$$

Case $\pi_{\mp}(a)=\hat{\pi}$. We put $a_{ \pm}^{\prime}=a_{ \pm}$and $\pi_{\mp}\left(a^{\prime}\right)=\pi$. Then by the previous theorem and Theorem 1 , we have

$$
\begin{aligned}
& \omega^{ \pm}\left(\mathscr{D}^{ \pm}, a, \pi\right) \leqq \omega^{ \pm}\left(\mathscr{D}^{ \pm}, a^{\prime}, \pi\right) \\
& \leqq \min _{x \in X} \omega^{ \pm}\left(\mathscr{D}_{x: \pi}^{ \pm}, a, \pi\right) \leqq \omega^{ \pm}\left(\mathscr{D}_{S \cdot \pi}^{ \pm}, a_{ \pm}, \pi\right) .
\end{aligned}
$$

Now our proof is completed.

\section{§ 7. Division Games over Impartial Spaces (1)}

In this section we deal with impartial spaces, after we introduce the Lehman's remark on games over regular spaces. First we define several terminologies on assignments.

Let $p \in \boldsymbol{N}$ and $N \in \overline{\boldsymbol{N}}$. Then an assignment $a$ of length $N$ is said to be $2 \cdot p^{+}$-periodic if

$$
a(i)=a\left(i^{\prime}\right) \text { iff }\left[\frac{i-1}{p}\right] \equiv\left[\frac{i^{\prime}-1}{p}\right] \bmod 2
$$

and $2 \cdot p^{-}$-periodic if 


$$
a(i)=a\left(i^{\prime}\right) \text { iff }\left[\frac{N-i}{p}\right] \equiv\left[\frac{N-i^{\prime}}{p}\right] \bmod 2 \text {, }
$$

where [] denotes the integral part of the entry. A $2 \cdot p^{ \pm}$-periodic assignment $a$ is said to be $2 \cdot p$-periodic in case $N \equiv 0 \bmod 2 p$ and alternate in case $p=1$. We understand that the sole assignment of length 0 is alternate.

Let $\mathscr{D}^{+}=\left(X, \chi^{+}\right)$be a regular division space with $|X| \neq 0$. Then there are two alternate assignments, say $a$ and $\wedge \circ a$, over it. Lehman [9] remarks that

$$
\omega^{+}\left(\mathscr{D}^{+}, a, \pi_{+}(a)\right) \geqq \omega^{+}\left(\mathscr{D}^{+}, \widehat{\wedge} \circ a, \pi_{+}(a)\right) .
$$

For a misère division space $\mathscr{D}^{-}=\left(X, \chi^{-}\right)$with $|X| \neq 0$, we have

$$
\omega^{-}\left(\mathscr{D}^{-}, a, \pi_{-}(a)\right) \geqq \omega^{-}\left(\mathscr{D}^{-}, \hat{\wedge} \circ a, \pi_{-}(a)\right) .
$$

These results follow immediately from Proposition 4 and Theorem 5.

From now on, in this section, every division space $\mathscr{D}^{*}$ is assumed to be impartial, namely, the index $\left|\mathscr{A}_{u t} \mathscr{D}^{*} / \mathscr{A}_{u t} \mathscr{D}^{*}\right|=2$. Then it is easy to see that $|X| \neq 0$.

Lemma 2. Let $\mathscr{D}^{*}$ be an impartial division space and a an assignment over it. Then we have

$$
\omega^{ \pm}\left(\mathscr{D}^{*}, \widehat{\wedge} a, \hat{\pi}\right)=\omega^{ \pm}\left(\mathscr{D}^{*}, a, \pi\right) .
$$

It is easy to prove this lemma.

Theorem 6. Let $\mathscr{D}^{*}=\left(X, \chi^{*}\right)$ be an impartial division space with $|X|$ even and $p$ a divisor of $|X| / 2$. Let a be a $2 \cdot p$-periodic assignment over $\mathscr{D}^{*}$. Then

$$
\omega^{+}\left(\mathscr{D}^{*}, a, \pi_{+}(a)\right)=1
$$

i.e.,

$$
\omega^{-}\left(\mathscr{D}^{*}, a, \pi_{-}(a)\right)=1 .
$$

Proof. We have

$$
\omega^{+}\left(\mathscr{D}^{*}, a, \pi_{+}(a)\right)
$$




$$
\begin{aligned}
& \geqq \omega^{+}\left(\mathscr{D}^{*}, a \circ s_{|X|}, \pi_{+}(a)\right) \\
& \geqq \cdots \ldots \ldots \ldots \ldots \ldots \ldots \ldots \ldots \\
& \geqq \omega^{+}\left(\mathscr{D}^{*}, a \circ s_{|X|}^{p}, \pi_{+}(a)\right)
\end{aligned}
$$

by Proposition 4. On the other hand $\mathscr{D}^{*}$ is impartial and $a \circ s_{|\mathrm{X}|}^{p}=$ ヘ。a. Therefore we easily obtain by Lemma 2

$$
\begin{aligned}
& \omega^{+}\left(\mathscr{D}^{*}, a \circ s_{|X|}^{p}, \pi_{+}(a)\right) \\
= & \omega^{+}\left(\mathscr{D}^{*}, a, \hat{\pi}_{+}(a)\right) \\
= & -\omega^{+}\left(\mathscr{D}^{*}, a, \pi_{+}(a)\right) .
\end{aligned}
$$

Now we have

$$
\omega^{+}\left(\mathscr{D}^{*}, a, \pi_{+}(a)\right) \geqq-\omega^{+}\left(\mathscr{D}^{*}, a, \pi_{+}(a)\right)
$$

i.e.,

$$
\omega^{+}\left(\mathscr{D}^{*}, a, \pi_{+}(a)\right)=1 \text {. }
$$

Theorem 7. Let $\mathscr{D}^{ \pm}=\left(X, \chi^{ \pm}\right)$be an impartial and also regular (misère) division space and let $a$ be a $2 \cdot p^{ \pm}$-periodic assignment of length $|X|$ for a positive integer $p$. Then we have

$$
\omega^{ \pm}\left(\mathscr{D}^{ \pm}, a, \pi_{ \pm}(a)\right)=1
$$

Proof. Assume that there exists an anti-automorphism $\sigma$ of $\mathscr{D}^{ \pm}$. Adding several negligible points to $\mathscr{D}^{ \pm}$we obtain a division space $\mathscr{D}^{\prime \pm}=\left(X^{\prime}, \chi^{\prime \pm}\right)$ such that $\left|X^{\prime}\right| \equiv 0 \bmod 2 p$, where there is a natural immersion from $\mathscr{D}^{ \pm}$to $\mathscr{D}^{\prime \pm}$. The anti-automorphism $\sigma$ is naturally extended to that over $\mathscr{D}^{\prime \pm}$ which fixes $X^{\prime}-X$ pointwisely. We denote by $a^{\prime}$ the $2 \cdot p$-periodic assignment with $\pi_{ \pm}\left(a^{\prime}\right)=\pi_{ \pm}(a)$. We have

$$
\omega^{ \pm}\left(\mathscr{D}^{ \pm}, a, \pi_{ \pm}(a)\right)=\omega^{ \pm}\left(\mathscr{D}^{\prime \pm}, a^{\prime}, \pi_{ \pm}\left(a^{\prime}\right)\right)=1
$$

by the previous theorem and Proposition 5. Thus our proof is completed.

Corollary $^{+}($Hein and Nash). In a usual Hex game, the starting player has a winning strategy.

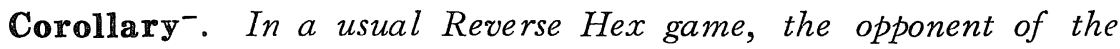


closing player has a winning strategy.

It is said that $O$. Winder also has obtained this corollary (see Gardner [5]). It seems that the properties $2 \cdot p^{+}$-periodic and $2 \cdot p^{-}$periodic in Theorem 7 can be replaced by the following properties respectively :

$$
\#\left\{i \leqq n \mid a(i)=\pi_{+}(a)\right\} \geqq \#\left\{i \leqq n \mid a(i)=\hat{\pi}_{+}(a)\right\} \quad{ }^{\forall} n
$$

and

$$
\#\left\{i \geqq n \mid a(i)=\pi_{-}(a)\right\} \geqq \#\left\{i \geqq n \mid a(i)=\hat{\pi}_{-}(a)\right\} \quad{ }^{\forall} n \text {. }
$$

However we shall give a counter example in the last section.

\section{§ 8. Division Games over Impartial Spaces (2)}

In this section we are going to show some detailed results over certain type of division spaces. We first introduce the following notion.

Let a division space $\mathscr{D}^{*}=\left(X, \chi^{*}\right)$ be fixed. Let $a$ be an assignment over it and $\pi$ a player. Then we put

$$
|a|_{\pi}^{+}=\#\left\{n \in N \mid a(i)=\pi \text { for }{ }^{\forall} i \leqq n\right\}
$$

and

$$
|a|_{\pi}^{-}=\#\left\{n \in N \mid a(i)=\pi \text { for }{ }^{\forall} i \geqq n\right\}
$$

Now we put

$$
Z_{\pi}^{ \pm}(a)=\left\{\left.S \subset X|\quad| S|=| a\right|_{\pi} ^{ \pm}, \omega^{ \pm}\left(\mathscr{D}_{S \cdot \pi}^{*}, a_{\mp|S|}, \pi\right)=1\right\} .
$$

Proposition 6. Let $\mathscr{D}^{*}$ be a division space and a an assignment over it. Then we have

$$
\begin{cases}(+) & Z_{\pi}^{+}(a) \neq \varnothing \text { iff } \omega^{+}\left(\mathscr{D}^{*}, a, \pi\right)=1 \\ (-) & Z_{\pi}^{-}(a) \neq \varnothing \text { implies } \omega^{-}\left(\mathscr{D}^{*}, a, \pi\right)=1 .\end{cases}
$$

This assertion is an immediate consequence of Theorem 1 .

Theorem 8. Let $p \in \mathbb{N}$. Let $\mathscr{D}^{ \pm}=\left(X, \chi^{ \pm}\right)$be a division space 
with $|X| \geqq 2 p$ having an anti-automorphism $\sigma=\left(\sigma^{x}\right.$, sgn $\left.\sigma\right)$, and let a be a $2 \cdot p^{ \pm}$-periodic assignment over $\mathscr{D}^{ \pm}$. Assume that there exist two subsets $A$ and $B$ of $X$ satisfying the following:

$$
A \cap B=\varnothing,|A|=|B|=p, \sigma^{x}(A)=A
$$

and

$$
X^{0}\left(\mathscr{D}_{B \cdot \pi_{ \pm}(a)}^{ \pm}\right) \supset A,
$$

where $X^{0}$ is the set of negligible points of the division space. Then

$$
Z_{\pi_{ \pm}(a)}^{ \pm}(a) \not \nexists A \text {. }
$$

Proof. By Theorem 1 and Theorem 5,

$$
\begin{aligned}
& \omega^{ \pm}\left(\mathscr{D}_{A \cdot \pi_{ \pm}(a)}^{ \pm}, a_{\mp p}, \pi_{ \pm}(a)\right) \\
& \leqq \omega^{ \pm}\left(\mathscr{D}_{(A \cup B) \cdot \pi_{ \pm}(a)}^{ \pm}, \quad\left(a_{\mp p}\right)_{ \pm p}, \pi_{ \pm}(a)\right) \\
& =\omega^{ \pm}\left(\mathscr{D}_{A}^{ \pm} \cdot \pi_{ \pm}(a)+B \cdot \pi_{ \pm}(a), \quad\left(a_{ \pm p}\right)_{\mp p}, \pi_{ \pm}(a)\right) \\
& \leqq \omega^{ \pm}\left(\mathscr{D}_{A \cdot t_{ \pm}(a)}^{ \pm}, a_{ \pm p}, \pi_{ \pm}(a)\right) \text {. }
\end{aligned}
$$

Here we have $a_{ \pm p}=\wedge \circ a_{\mp p}$. Then by Lemma 2

$$
\begin{aligned}
& \omega^{ \pm}\left(\mathscr{D}_{A \cdot \pi_{ \pm}(a)}^{ \pm}, a_{ \pm p}, \pi_{ \pm}(a)\right) \\
= & \omega^{\mp}\left(\mathscr{D}_{A \cdot \pi_{ \pm}(a)}^{ \pm}, a_{\mp p}, \pi_{ \pm}(a)\right) .
\end{aligned}
$$

Now we obtain

$$
\begin{aligned}
& \omega^{ \pm}\left(\mathscr{D}_{A \cdot \pi_{ \pm}(a)}^{ \pm}, a_{\mp p}, \pi_{ \pm}(\mathrm{a})\right) \\
\leqq & \omega^{\mp}\left(\mathscr{D}_{A \cdot \pi_{ \pm}(a)}^{ \pm}, a_{\mp p}, \pi_{ \pm}(a)\right) .
\end{aligned}
$$

Therefore

$$
\omega^{ \pm}\left(\mathscr{D}_{A}^{ \pm} \cdot_{ \pm}(a), a_{\mp p}, \pi_{ \pm}(a)\right)=-1
$$

i. e.,

$$
Z_{\pi_{ \pm}(a)}^{ \pm}(a) \nexists A
$$

Corollary $^{+}(\mathrm{Beck})$. In a usual $n \times n$ Hex game for $n \geqq 2$, the starting player has no winning strategy making the first move to one of the acute corners.

Corollary-. In a usual $n \times n$ Reverse Hex game for $n \geqq 2$, the opponent of the closing player has no winning strategy reserving a 
fixed acute corner for the closing player.

Corollary (Evans). In a usual $n \times n$ Reverse Hex game for an even $n$, the starting player has a winning strategy making the first move to one of acute corners.

These corollaries follow easily, since an acute corner can be made negligible if one occupies a certain point adjacent to it, and since $\pi_{+}\left(a_{-}\right)=\pi_{-}(a)=\pi_{-}\left(a_{+}\right)$for an alternate assignment $a$ of even length.

\section{$\S 9 . \quad$ Division Games and Block Designs}

Let $(t, v, k, \lambda)$ be a 4-tupple of non-negative integers such that $v \geqq k \geqq t$ and $\boldsymbol{D}=(X, \mathfrak{B}, I)$ a triplet of finite sets $X, \mathfrak{B}$ and a subset $I$ of $X \times \mathfrak{B}$. Suppose that $D$ satisfies the following conditions :

$$
\begin{aligned}
& |X|=v \\
& \#\{x \in X \mid(x, B) \in I\}=k \quad{ }^{\forall} B \in \mathfrak{B}
\end{aligned}
$$

and

$$
\#\left\{B \in \mathfrak{B} \mid(y, B) \in I,{ }^{\forall} y \in Y\right\}=\lambda
$$

for any subset $Y$ consisting of $t$ points of $X$. Then $D$ is called a block design of parameter $(t, v, k, \lambda) . \mathbb{D}$ is called also a $t$-design of parameter $(v, k, \lambda)$ or more simply a $t-(v, k, \lambda)$ design. An element $x$ of $X$ is called a point and one $B$ of $\mathfrak{B}$ is called a block. As is easily seen, a $t$-design is also a $t^{\prime}$-design for $t^{\prime} \leqq t$. A $2-(v, 3$, $\lambda$ ) design is called a triple system. It is known that there exists a triple system of given parameter $(v, \lambda)$ if and only if both $\lambda \cdot \frac{v-1}{k-1}$ and $\lambda \cdot \frac{v-1}{k-1} \cdot \frac{v-2}{k-2}$ are integers (cf. Hall [7] and Hanani [8]).

Let $\boldsymbol{D}=(X, \mathfrak{B}, I)$ be a block design. For any block $B$, we denote by $(B)$ the set $\{x \in X \mid(x, B) \in I\}$. Then $D$ is said to have no repetition if $\left(B_{1}\right)=\left(B_{2}\right)$ implies $B_{1}=B_{2}$. If $D$ has no repetition, then $(B)$ is identified with $B$. Let $\mathbb{D}^{\prime}=\left(X^{\prime}, \mathfrak{B}^{\prime}, I^{\prime}\right)$ be another block design. Then $\boldsymbol{D}$ and $\mathbb{D}^{\prime}$ are said to be isomorphic if there exists bijections $f^{X}: X \rightarrow X^{\prime}$ and $f^{\mathfrak{P}}: \mathfrak{B} \rightarrow \mathfrak{B}^{\prime}$ such that 


$$
f^{x} \times f^{\mathfrak{B}}(I)=I^{\prime}
$$

Let $\pi$ be a player, $v$ and $k$ non-negative integers such that $v \geqq k$. We set

$$
\mathfrak{D}^{\pi_{k} k}=\left\{\mathfrak{D} \in \mathfrak{D}_{X}\left|\chi^{*}(\mathfrak{D}, \pi)=1,\right| \mathfrak{D}^{-1}(\pi) \mid=k\right\}
$$

for any division space $\mathscr{D}^{*}=\left(X, \chi^{*}\right)$. Let $\mathscr{D}_{1}^{*}\left(X_{1}, \chi_{1}^{*}\right)$ and $\mathscr{D}_{2}^{*}=$ $\left(X_{2}, \chi_{2}^{*}\right)$ be division spaces satisfying $\left|X_{1}\right|=\left|X_{2}\right|=v$. Then $\mathscr{D}_{1}^{*}$ and $\mathscr{D}_{2}^{*}$ are said to be $(\pi, k)$-equivalent if there exists a bijection $f^{x}$ : $X_{1} \rightarrow X_{2}$ such that

$$
f^{\mathfrak{B}}\left(\mathfrak{D}_{2}^{\pi, k}\right)=\mathfrak{D}_{1}^{\pi, k},
$$

where $f^{\mathfrak{g}}\left(\mathfrak{D}_{2}\right)=\mathfrak{D}_{2} \circ f^{\mathrm{X}}$ for $\mathfrak{D}_{2} \in \mathfrak{D}_{2}^{\pi, k}$. We obtain a 1: 1-correspondence between $(\pi, \mathrm{k})$-equivalence class of division space $\mathscr{D}^{*}=\left(X, \chi^{*}\right)$ satisfying $|X|=v$ and isomorphism class of $0-(v, k, \lambda)$ designs without repetition given as follows:

$$
\left[\left(X, \chi^{*}\right)\right] \rightarrow\left[\left(X, \mathfrak{P}^{\pi, k}, I\right)\right]
$$

where $(x, \mathfrak{D}) \in I$ if and only if $\mathfrak{D}(x)=\pi$,

$$
\left[\left(X, \chi^{*}\right)\right] \leftarrow[(X, \mathfrak{B}, I)]
$$

where $\chi^{*}(\delta, \pi)=1$ if and only if $\delta^{-1}(\pi)$ forms a block. We call this correspondence the $(\pi, k)$-correspondence.

Theorem 9. Let $\pi$ be a player, $(t, v, \lambda)$ a triplet of non-negative integers such that $v \geqq t+\lambda+1$ and $\mathbb{D}=(X, \mathfrak{B}, I)$ a $0-(v, t+1, \lambda)$ design without repetition satisfying

$$
|\mathfrak{B}| \geqq \lambda \cdot\left(\begin{array}{c}
v \\
t
\end{array}\right) /(t+1) .
$$

We denote by $\mathscr{D}^{*}=\left(X, \chi^{*}\right)$ a division space of the class $(\pi, t+1)$ corresponding to the class $[\mathbb{D}]$. Then $\mathbb{D}$ is a $t$ - $(v, t+1, \lambda)$ design if and only if

$$
\omega^{+}\left(\mathscr{D}^{*}, a_{0}, \pi\right)=-1
$$

where the assignment $a_{0}$ of length $v$ is defined by

$$
a_{0}(i)=\pi \quad \text { iff } \quad i \leqq t \quad \text { or } \quad i=t+\lambda+1 .
$$


Proof. First assume $\omega^{+}\left(\mathscr{D}^{*}, a, \pi\right)=-1$. Suppose that $\pi$ has occupied a set $Y$ consisting of $t$ points of $X$. Then $\hat{\pi}$ is to win if he occupies a suitable $\lambda$ points say $x_{1}, \cdots, x_{\lambda}$ of $X-Y$ for him. Now any block $B$ containing $Y$ coincides with some $Y \cup\left\{x_{i}\right\}$. Thus for any subset $Y$ consisting of $t$ points of $X$, we have

$$
\#\left\{B \in \mathfrak{B} \mid(y, B) \in I \quad{ }^{\forall} y \in Y\right\} \leqq \lambda .
$$

Summing up, we obtain

$$
\left(\begin{array}{c}
t+1 \\
t
\end{array}\right) \cdot|\mathfrak{B}| \leqq \lambda \cdot\left(\begin{array}{c}
v \\
t
\end{array}\right)
$$

On the other hand we have assumed $|\mathfrak{B}| \geqq \lambda \cdot\left(\begin{array}{c}v \\ t\end{array}\right) /(t+1)$. Then $\#\left\{B \in \mathfrak{B} \mid(y, B) \in I{ }^{\forall} y \in Y\right\}$ must be exactly $\lambda$ for any subset $Y$ consisting of $t$ points of $X$. Namely, $D$ is a $t$ - $(v, t+1, \lambda)$ design. Next assume that $\boldsymbol{D}$ is a $t-(\nu, t+1, \lambda)$ design. Suppose that $\pi$ has occupied a set $Y$ consisting of $t$ points of $X$. Then $\hat{\pi}$ finds $\lambda$ blocks $B_{1}, \cdots, B_{\lambda}$ containing $Y$, and occupies $\cup_{i=1}^{\lambda}\left(B_{i}-Y\right)$. After it $\pi$ finds no way to win.

Proposition 7. We have a $2(6,3,2)$ design without repetition, which is defined in the following way. Let $\tilde{X}$ be the set of vertices of an icosahedron and $\alpha$ the antipodal permutation on $\tilde{X}$. We denote by $X$ the orbit space with respect to the group $\langle\alpha\rangle$ and by $\langle\alpha\rangle \backslash$ the natural mapping from $\tilde{X}$ to $X$. Let $\mathfrak{B}$ be the family of subsets $B$ consisting of three elements of $X$ such that $(\langle\alpha\rangle)^{-1}(X-B)$ consists of vertices of an antipodal pair of 2-simplices of the relevant icosahedron. We define as usual a subset $I$ of $X \times \mathfrak{B}$ by

$$
(x, B) \in I \text { iff } x \in B \text {. }
$$

Then $\boldsymbol{D}=(X, \mathfrak{B}, I)$ forms a 2-(6, 3, 2) design without repetition. Therefore fixing a player $\pi$, we have

$$
\omega^{+}\left(\mathscr{D}^{*}, a_{0}, \pi\right)=-1
$$

for a division space of the class $(\pi, 3)$-corresponding to $[D]$ and the assignment $a_{0}$ of length 6 such that

$$
a_{0}(i)=\pi \text { iff } i=1,2,5 \text {. }
$$


Proof. We easily see that $|\mathfrak{B}|=10$. By Theorem 9 we have to show only that $D$ is a $2-(6,3,2)$ design without repetition. For any pair $(x, y)$ of distinct elements of $X,(\langle\alpha\rangle \backslash)^{-1}(\{x, y\})$ is the set of vertices of an antipodal pair of 1 -simplices. Therefore $D$ is a block design of desired parameter. Now our proof is complete.

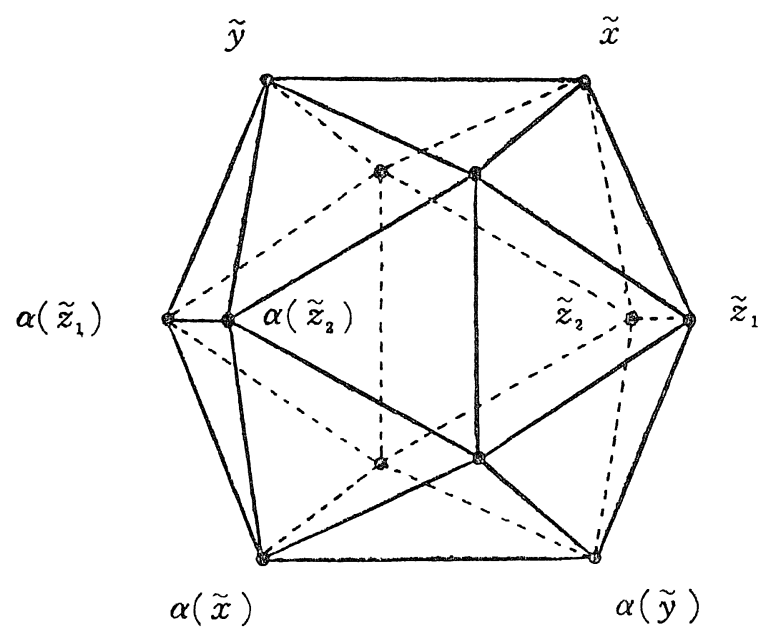

Figure 2

The above $\mathscr{D}^{*}$ is $(\pi, 3)$ equivalent to a minimal example with respect to $|X|$, of a regular impartial division space $\mathscr{D}^{+}=\left(X, \chi^{+}\right)$such that

$$
\omega^{+}\left(\mathscr{D}^{+}, a_{0}, \pi\right)=-1
$$

for an assignment $a_{0}$ of length $|X|$ satisfying

$$
\#\left\{i \leqq n \mid a_{0}(i)=\pi\right\} \geqq \#\left\{i \leqq n \mid a_{0}(i)=\hat{\pi}\right\} \quad{ }^{\forall} n .
$$

As is also known, there exist the sole 4-(11,5,1) design $\mathbb{W}_{11}$ and the sole 5 - $(12,6,1)$ design $W_{12}$, whose automorphism groups are Mathieu groups $M_{11}$ and $M_{12}$. These $W_{11}$ and $W_{12}$ also form remarkable examples of block designs characterized in a game theoretical argument of Theorem 9.

\section{References}

[1] Beck, A., Bleicher, M. and Crowe, Excursion into Mathematics, Worth Publisher, Inc., New York, 1969.

[2] Evans, R., A winning opening in Reverse Hex, J. Recreational Math., 7, (1974), 189-192. 
[3] Even, S., and Tarjan, R. E., A combinatorial problem which is complete in polynomial space, Proc. Seventh Annual A. C. M. Symposium on Theory of Computing, May, 1975.

[4] Gardner, M., The Scientific American Book of Mathematical Puzzles \& Diversions, Simon and Schuster, Inc., New York, 1959.

[5] Gardner, M., Mathematical games, Scientific American, June 1975, 106-111.

[6] Greenberg, P. J., Mathieu Groups, Courant Institute of Math. Science, New York Univ.

[7] Hall, M. Jr., Combinato rial Theory, Blaisdell, 1968.

[8] Hanani, H., The existence and construction of balanced imcomplete block designs, Ann. Math. Statist., 32 (1961), 361-386.

[9] Lehman, A., A solution of the Shannon switching game, J. Soc. Ind. Appl. Math., 12, (1964).

[10] Sato, M., Representation theory of division spaces and division games, private communication, July, 1975.

[11] Yamasaki, Y., A generalization of Hex (Nash game) (in Japanese), Kokyu roku, RIMS, Kyoto Univ., 263, 1976. 